\title{
VISUAL CONTROL OF ROBOTIC MANIPULATORS: DESIGNING A SIMPLIFIED STABILIZING CONTROLLER
}

\author{
L. Bascetta P. Rocco ${ }^{1}$ \\ Politecnico di Milano, Dip. Elettronica e Informazione, Piazza \\ Leonardo da Vinci, 32, 20133, Milano, Italy \\ \{bascetta,rocco\}@elet.polimi.it
}

\begin{abstract}
An Image Based Visual Servoing controller for the tip trajectory tracking of rigid manipulators is considered in this paper. Inspired by the task space robot control, an approach to design a visual control law, that takes into account the manipulator dynamics, is proposed. Moreover, to avoid an excessive computational burden, two simplified visual control laws are introduced supported by rigorous proofs of stability based on Lyapunov theory. Simulation results show the effectiveness of these simplified controllers. Copyright $@ 2005$ IFAC
\end{abstract}

Keywords: Visual servoing, task space control, robotic manipulators, Lyapunov theory.

\section{INTRODUCTION}

When high performance position control of a manipulator is considered, visual feedback can considerably improve the precision of the end effector positioning. Visual information, unlike standard position sensors yields a direct measurement of the tip position, avoiding estimation errors due to a rough knowledge of the physical parameters characterizing the mechanical chain (e.g. gear train elasticity, friction, gear backlash). For these reasons the interest towards the control of robots by means of visual feedback (so called visual servoing (Hutchinson et al., 1996)) has been increasing in recent years.

The present work deals with the eye-in-hand Image Based Visual Servoing of rigid manipulators, with an approach that is inspired by the well-known task space robot control (Sciavicco and Siciliano, 2000).

While suffering from some known (Chaumette, 1998) limitations, the IBVS approach is still an attractive solution as it computes the feedback control directly from the visual information, and does not require any further knowledge of the physical parameters of the

\footnotetext{
1 This work has been supported by ASI (Italian Space Agency) under contract number ASI I/R/120/02.
}

manipulator. Thus, it effectively represents a suitable approach to design a high performance position controller.

On the other hand, by analogy with positional task space control, at least two visual control structures can be derived: a $P D$ controller based on the transpose Jacobian control law, as in (Kelly, 1996; Kelly et al., 2000), and a computed torque control law. Although the $P D$ controller proposed in (Kelly et $a l ., 2000)$ is particularly simple, as it does not require the calculation of the inverse of the Jacobian and is independent of the robot inertia and Coriolis matrices, it depends on the design of two gain matrices that cannot be clearly related to the closed loop desired dynamics.

A fully linearizing task space control law is first derived in the present paper. Then, in order to reduce the computational burden, two simplified visual controllers are presented, where computation of the inverse and time derivative of the Jacobian and estimation of the target acceleration, required by the computed torque algorithm, are avoided. Furthermore the closed loop stability of the system under the simplified control laws is proven invoking the Lyapunov theory. 


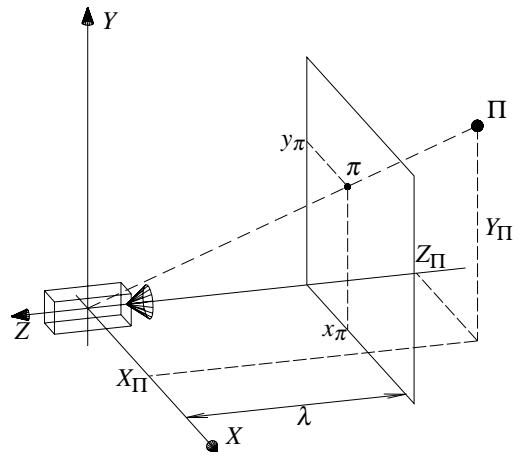

Figure 1. A simple camera model

Finally, simulation results are presented, focusing on the model of a two link planar manipulator: the trajectory tracking performance of the simplified visual controllers, when an increasing acceleration of the target is considered, are investigated.

The paper is organized as follows. In Section 2 the dynamic models of the camera and of a rigid manipulator are described. The control problem is formulated in Section 3 and a solution, based on the task space control technique, is presented. In Section 4 two simplified control laws are proposed and a stability analysis, based on the Lyapunov theory, of the closed loop system under these simplified controllers is shown. Finally, Section 5 presents a simulation based on a two link planar manipulator. Conclusions are given in Section 6.

\section{ROBOTIC SYSTEM MODEL}

\subsection{Manipulator dynamics}

The dynamic model of a rigid manipulator with $n$ links can be written in the following form

$$
\mathbf{M}(\mathbf{q}) \ddot{\mathbf{q}}+\mathbf{f}(\mathbf{q}, \dot{\mathbf{q}})+\mathbf{g}(\mathbf{q})=\mathbf{u}
$$

where $\mathbf{M}$ is the inertia matrix, $\mathbf{q}=\left(q_{1} q_{2} \ldots q_{n}\right)^{T}$ is the vector of joint variables, $\mathbf{f}$ is the vector containing Coriolis and centrifugal terms, $\mathbf{g}$ is the vector of gravitational torques and $\mathbf{u}=\left(\begin{array}{llll}\tau_{1} & \tau_{2} & \ldots & \tau_{n}\end{array}\right)^{T}$ is the vector of control torques applied at the $n$ joints.

\subsection{Camera model}

Assuming that the camera is modelled by perspective projection (Hutchinson et al., 1996), a point ${ }^{c} \Pi=$ $\left[\begin{array}{lll}X & Y & Z\end{array}\right]^{T}$, whose coordinates are expressed with respect to the camera frame $c$ (Fig. 1), will project onto a point $\pi=\left[\begin{array}{ll}x y & y\end{array}\right]^{T}$ of the image plane given by

$$
\left[\begin{array}{l}
x \\
y
\end{array}\right]=-\alpha \frac{\lambda}{Z}\left[\begin{array}{l}
X \\
Y
\end{array}\right]
$$

where $\lambda$ is the focal length, $\alpha$ is the scaling factor in pixel per meter due to camera spatial sampling and $Z<0$ according to the chosen orientation of the camera frame.

Let us define an image feature as any structural feature that can be extracted from an image and an image feature parameter as any real-valued quantity that can be calculated from one or more of them (Hutchinson et al., 1996).

Consider an eye-in-hand camera system and a moving target: a linear differential relation between the relative motion of the target, with respect to the camera, and the image feature motion can be stated as follows

$$
\dot{\xi}=J_{\text {image }}\left(\mathbf{r}, \mathbf{p}_{t}\right)\left[\begin{array}{c}
\dot{\mathbf{r}} \\
\dot{\mathbf{p}}_{t}
\end{array}\right]
$$

where $\xi=\left[\begin{array}{llll}\xi_{1} & \ldots & \xi_{f}\end{array}\right]^{T}$ is a vector of $f$ image feature parameters, $\mathbf{r}$ the vector of end effector coordinates with respect to any parametrization of the task space and $\mathbf{p}_{t}$ the vector of target coordinates with respect to the robot frame. The matrix $J_{\text {image }} \in \mathfrak{R}^{f \times(s+\mathscr{T})}$, where $s$ and $\mathscr{T}$ are the dimensions of the task space and the number of degrees of freedom of the target, respectively, is the so-called image Jacobian

$$
J_{\text {image }}\left(\mathbf{r}, \mathbf{p}_{t}\right)=\left[\begin{array}{ll}
\frac{\partial \xi}{\partial \mathbf{r}} & \underbrace{\frac{\partial \xi}{\partial \mathbf{p}_{t}}}_{J_{R}}
\end{array}\right]
$$

The analytical expression of the image Jacobian depends on the actual parameters chosen to describe the considered image features (see (Kelly et al., 2000) for the analytical expressions of the main image Jacobians).

\section{A VISUAL SERVO CONTROLLER}

Let $\xi \in \mathfrak{R}^{f}$ be a generic feature parameter vector and $J(\mathbf{q}, \xi, \mathbf{Z})$ the corresponding Jacobian, $\xi_{d} \in \mathfrak{R}^{f}$ the desired feature parameter vector, which is assumed to be constant, and define the image feature error $\tilde{\xi} \in \mathfrak{R}^{f}$ as $\tilde{\xi}=\xi_{d}-\xi$.

The control problem can be formulated as follows: design a controller that computes the robot torques $\mathbf{u}$ in such a way that the image feature error $\tilde{\xi}$ vanishes.

The following assumptions are enforced to ensure that the control problem is solvable and the task space inverse dynamic approach can be applied:

A1 for any target position there exists a robot joint configuration for which the feature error vanishes, i.e. $\forall \Pi \exists \mathbf{q}_{d}: \xi_{d}=\xi\left(\mathbf{q}_{d}, \Pi\right)$;

A2 the desired joint configuration $\mathbf{q}_{d}$ is an isolated solution of $\xi(\mathbf{q}, \Pi)=\xi_{d} ;$

A3 the Jacobian $J(\mathbf{q}, \xi(\mathbf{q}, \Pi), \mathbf{Z}(\mathbf{q}, \Pi))$ is continuously differentiable with respect to each entry of $\mathbf{q}$ and $\Pi$.

The controller design is based on the model of the manipulator and is inspired by the well-known task space inverse dynamic robot control (Sciavicco and Siciliano, 2000). If a feedback control of the form

$$
\mathbf{u}(\mathbf{q}, \dot{\mathbf{q}})=\mathbf{f}_{1}(\mathbf{q}, \dot{\mathbf{q}})+\mathbf{M}(\mathbf{q}) \mathbf{v}
$$

is designed, providing a compensation of the nonlinear terms in $\mathbf{f}_{1}$, the robot model (1) reduces to a double integrator system $\ddot{\mathbf{q}}=\mathbf{v}$. 
Consider now the differential relation introduced in the previous section

$$
\dot{\xi}=J(\mathbf{q}, \xi, Z)\left[\begin{array}{c}
\dot{\mathbf{q}} \\
\dot{\Pi}
\end{array}\right]
$$

and its time derivative

$$
\ddot{\xi}=J(\mathbf{q}, \xi, Z)\left[\begin{array}{c}
\ddot{\mathbf{q}} \\
\ddot{\Pi}
\end{array}\right]+\dot{J}(\mathbf{q}, \dot{\mathbf{q}}, \xi, \dot{\xi}, Z, \dot{Z})\left[\begin{array}{c}
\dot{\mathbf{q}} \\
\dot{\Pi}
\end{array}\right]
$$

If a control $\mathbf{v}$ as

$$
\begin{array}{r}
\mathbf{v}=J_{R}(\mathbf{q}, \xi, Z)^{-1}\left[K_{D} \dot{\tilde{\xi}}+K_{P} \tilde{\xi}-J_{T}(\mathbf{q}, \xi, Z) \ddot{\Pi}\right. \\
\left.-\dot{J}_{R}(\mathbf{q}, \dot{\mathbf{q}}, \xi, \dot{\xi}, Z, \dot{Z}) \dot{\mathbf{q}}-\dot{J}_{T}(\mathbf{q}, \dot{\mathbf{q}}, \xi, \dot{\xi}, Z, \dot{Z}) \dot{\Pi}\right]
\end{array}
$$

is chosen, assuming the desired feature vector constant, the double integrator system gives rise to the following equation in the feature errors

$$
\ddot{\tilde{\xi}}+K_{D} \dot{\tilde{\xi}}+K_{P} \tilde{\xi}=\mathbf{0}
$$

where $K_{P}$ and $K_{D}$ can be chosen as diagonal positive definite matrices. The rate of convergence of the feature errors can thus be selected arbitrarily, assigning the frequencies $\omega_{i}$ and the damping factors $\zeta_{i}$ that characterize the pair of eigenvalues corresponding to each feature error in (3) and picking matrices $K_{P}$ and $K_{D}$ as follows

$$
\begin{aligned}
& K_{P}=\operatorname{diag}\left(\omega_{1}^{2} \ldots \omega_{f}^{2}\right) \\
& K_{D}=\operatorname{diag}\left(2 \zeta_{1} \omega_{1} \ldots 2 \zeta_{f} \omega_{f}\right)
\end{aligned}
$$

\section{A SIMPLIFIED VISUAL CONTROLLER}

The visual control law (2) can be rather difficult to implement as it requires the calculation of the inverse and of the time derivative of the Jacobian matrix. A simplified controller implementation can be derived from (2) as follows

$$
\mathbf{v}=J_{R}(\mathbf{q}, \xi, Z)^{\#}\left[K_{D} \dot{\tilde{\xi}}+K_{P} \tilde{\xi}-J_{T}(\mathbf{q}, \xi, Z) \ddot{\Pi}\right]
$$

where the inverse of the Jacobian is replaced by $J_{R}(\mathbf{q}, \xi, Z)^{\#}$, a suitable pseudo inverse, and its time derivative is neglected. Moreover, the computation of the target acceleration $\ddot{\Pi}$, that can be achieved through computer vision techniques (Horn, 1986), might be considered an excessive burden. A further simplification of the control law (4), obtained by neglecting the target acceleration, yields

$$
\mathbf{v}=J_{R}(\mathbf{q}, \xi, Z)^{\#}\left[K_{D} \dot{\tilde{\xi}}+K_{P} \tilde{\xi}\right]
$$

This Section will be devoted to the stability analysis of the closed loop under the simplified visual control laws (4) and (5). Using these control laws, the feature error dynamic (3) becomes

$$
\dot{\mathbf{e}}=A \mathbf{e}-B \Delta B^{T} A \mathbf{e}+B \mathbf{d}
$$

where

$$
\begin{aligned}
A & =\left[\begin{array}{cc}
\mathbf{0}_{f} & I_{f} \\
-K_{P} & -K_{D}
\end{array}\right] \quad B=\left[\begin{array}{l}
\mathbf{0}_{f} \\
I_{f}
\end{array}\right] \\
\Delta & =I_{f}-J_{R}(\mathbf{q}, \xi, Z) J_{R}(\mathbf{q}, \xi, Z)^{\#}
\end{aligned}
$$

and

$$
\mathbf{e}=\left[\begin{array}{c}
\tilde{\xi} \\
\dot{\tilde{\xi}}
\end{array}\right] \quad \mathbf{d}=-\dot{J}(\mathbf{q}, \dot{\mathbf{q}}, \xi, \dot{\xi}, Z, \dot{Z})\left[\begin{array}{c}
\dot{\mathbf{q}} \\
\dot{\Pi}
\end{array}\right]
$$

if the control law (4) is used, or

$$
\mathbf{d}=-\dot{J}(\mathbf{q}, \dot{\mathbf{q}}, \xi, \dot{\xi}, Z, \dot{Z})\left[\begin{array}{c}
\dot{\mathbf{q}} \\
\dot{\Pi}
\end{array}\right]-J_{T}(\mathbf{q}, \xi, Z) \ddot{\Pi}
$$

if the control law (5) is used.

The following assumptions are adopted.

Assumption 1. A damped least squares inverse of the Jacobian $J_{R}$ (Nakamura and Hanafusa, 1986), (Wampler, 1986) is adopted, i.e.

$$
J_{R}^{\#}=\left(J_{R}^{T} J_{R}+\varsigma^{2} I\right)^{-1} J_{R}^{T}
$$

where $\varsigma$ is the damping factor.

Assumption 2. All the feature errors have the same closed loop dynamics, i.e.

$$
K_{P}=\omega_{n}^{2} I_{f} \quad K_{D}=2 \zeta \omega_{n} I_{f}
$$

Assumption 3. The following relation holds for all $\mathbf{q}$ and $\Pi$ along the considered trajectory (Wampler, 1986)

$$
\|\Delta\| \leq \gamma=\frac{\varsigma^{2}}{\varsigma^{2}+\sigma_{R_{m}}^{2}}
$$

where $\sigma_{R_{m}}$ is the minimum of the smallest singular value of the Jacobian matrix $J_{R}$ along the considered robot and target trajectory.

Assumption 4. Define $N_{i}(\mathbf{q}, \Pi)$ as

$$
N_{i}(\mathbf{q}, \Pi)=\left[\begin{array}{ll}
\frac{\partial \mathbf{j}_{i}}{\partial \mathbf{q}} & \frac{\partial \mathbf{j}_{i}}{\partial \Pi}
\end{array}\right]
$$

being $\mathbf{j}_{i}^{T}$ the $i$ th row of matrix $J(\mathbf{q}, \xi(\mathbf{q}, \Pi), \mathbf{Z}(\mathbf{q}, \Pi))$. Vector

$$
\mathbf{d}_{1}=-\dot{J}(\mathbf{q}, \dot{\mathbf{q}}, \xi, \dot{\xi}, Z, \dot{Z})\left[\begin{array}{c}
\dot{\mathbf{q}} \\
\dot{\Pi}
\end{array}\right]
$$

can be bounded as follows

$$
\left\|\mathbf{d}_{1}\right\| \leq \frac{v}{\sigma_{m}^{2}}\|\dot{\xi}\|^{2}
$$

where $\sigma_{m}$ is the minimum of the smallest singular value of the Jacobian matrix $J$ along the considered robot and target trajectory and

$$
v=\max _{\mathbf{q}, \Pi} \sqrt{\sum_{i=1}^{f}\left\|N_{i}(\mathbf{q}, \Pi)\right\|^{2}}
$$

being $\left\|N_{i}\right\|$ the largest singular value of matrix $N_{i}$ for all $\mathbf{q}$ and $\Pi$ along the considered trajectory.

Assumption 5. Vector

$$
\mathbf{d}_{2}=-J_{T}(\mathbf{q}, \xi, Z) \ddot{\Pi}
$$

can be bounded as follows

$$
\left\|\mathbf{d}_{2}\right\| \leq \sigma_{T_{M}} \chi_{M}
$$

where $\sigma_{T_{M}}$ is the maximum of the largest singular value of the Jacobian matrix $J_{T}$ along the considered robot and target trajectory and

$$
\chi_{M}=\max _{t}\|\ddot{\Pi}(t)\|
$$


Note that from the structure of matrices $A, K_{P}, K_{D}$ and Assumption 2 it can be easily concluded that system (6) is actually composed of $f$ independent subsystems, with state matrices

$$
\mathrm{A}=\left[\begin{array}{cc}
0 & 1 \\
-\omega_{n}^{2} & -2 \zeta \omega_{n}
\end{array}\right] \quad \mathrm{B}=\left[\begin{array}{l}
0 \\
1
\end{array}\right]
$$

Matrices $(A, B)$ of the whole system can be rearranged, through a suitable change of coordinates, as follows

$$
\mathscr{A}=b \operatorname{diag}(\mathrm{A} \ldots \mathrm{A}) \quad \mathscr{B}=\left(\mathscr{B}_{1}^{T} \ldots \mathscr{B}_{f}^{T}\right)^{T}
$$

where the symbol $b$ diag denotes a block diagonal matrix and

$$
\mathscr{B}_{i}=\left[\begin{array}{lll}
\mathbf{0}_{2, i-1} & \mathrm{~B} & \mathbf{0}_{2, f-i}
\end{array}\right]
$$

Consider a scalar $\beta>0$ and assume that parameters $\omega_{n}$ and $\zeta$ are such that

$$
\omega_{n}^{2}=\beta^{2} \kappa_{01} \quad 2 \zeta \omega_{n}=\beta \kappa_{02}
$$

with

$$
\kappa_{02}>2 \quad \kappa_{01}>\kappa_{02}-1
$$

Let

$$
A_{o}=\left[\begin{array}{cc}
0 & 1 \\
-\kappa_{01} & -\kappa_{02}
\end{array}\right]
$$

and $\mathrm{P}_{o}$ is the solution of the algebraic Riccati equation

$$
\begin{aligned}
\left(\mathrm{A}_{o}+I_{2}\right)^{T} \mathrm{P}_{o}+\mathrm{P}_{o}\left(\mathrm{~A}_{o}+I_{2}\right) & +\gamma^{2} \mathrm{P}_{o} \mathrm{BB}^{T} \mathrm{P}_{o} \\
& +\mathrm{A}_{o}^{T} \mathrm{BB}^{T} \mathrm{~A}_{o}=0
\end{aligned}
$$

The following stability results can be stated:

Theorem 1. Consider the simplified visual control law

$$
\mathbf{v}=J_{R}(\mathbf{q}, \xi, Z)^{\#}\left[K_{D} \dot{\tilde{\xi}}+K_{P} \tilde{\xi}-J_{T}(\mathbf{q}, \xi, Z) \ddot{\Pi}\right]
$$

where $J_{R}(\mathbf{q}, \xi, Z)^{\#}$ is a damped least squares inverse of the Jacobian, as stated in Assumption 1, and $K_{P}$ and $K_{D}$ are gain matrices that satisfy Assumption 2.

Choose the values of the parameters $\kappa_{01}$ and $\kappa_{02}$ in such a way that (8) holds and

$$
\left\|\mathrm{B}^{T} \mathrm{~A}_{\circ}\left[s I-\left(\mathrm{A}_{o}+I_{2}\right)\right]^{-1} \mathrm{~B}\right\|_{\infty}<\gamma^{-1}
$$

Let $\mathrm{P}_{o}$ be a positive definite solution of (9) and

$$
\tilde{\beta}=\beta /\left\|\mathrm{P}_{o}^{1 / 2} \mathrm{~B}\right\|
$$

The origin of the state space $(\tilde{\xi}, \dot{\tilde{\xi}})$ is an asymptotically stable equilibrium point whose region of attraction includes the region

$$
\sum_{i=1}^{f}\left[\begin{array}{c}
\tilde{\xi}_{i} \\
\dot{\tilde{\xi}}_{i}
\end{array}\right]^{T} \mathbf{B} \mathrm{P}_{o} \mathbf{B}\left[\begin{array}{c}
\tilde{\xi}_{i} \\
\dot{\tilde{\xi}}_{i}
\end{array}\right]<\rho^{2}
$$

where $\mathbf{B}=\operatorname{diag}(\beta, 1)$ and $\rho=\frac{\sigma_{m}^{2}}{v} \tilde{\beta}$.

Theorem 2. Consider the simplified visual control law

$$
\mathbf{v}=J_{R}(\mathbf{q}, \xi, Z)^{\#}\left[K_{D} \dot{\tilde{\xi}}+K_{P} \tilde{\xi}\right]
$$

where $J_{R}(\mathbf{q}, \xi, Z)^{\#}$ is a damped least squares inverse of the Jacobian, as stated in Assumption 1, and $K_{P}$ and
$K_{D}$ are gain matrices that satisfy Assumption 2.

Choose the values of the parameters $\kappa_{01}$ and $\kappa_{02}$ in such a way that (8) holds and

$$
\left\|\mathrm{B}^{T} \mathrm{~A}_{\circ}\left[s I-\left(\mathrm{A}_{o}+I_{2}\right)\right]^{-1} \mathrm{~B}\right\|_{\infty}<\gamma^{-1}
$$

Let $\mathrm{P}_{o}$ be a positive definite solution of (9) and

$$
\tilde{\beta}=\beta /\left\|\mathrm{P}_{o}^{1 / 2} \mathrm{~B}\right\|
$$

Then, if the following condition holds

$$
\tilde{\beta}>\frac{2}{\sigma_{m}} \sqrt{\nu \sigma_{T_{M}} \chi_{M}}
$$

there exist two convex regions, $\mathscr{S}_{1}$ and $\mathscr{S}_{2}$, of the state space such that the origin of the state space belongs to $\mathscr{S}_{1}$, being $\mathscr{S}_{1} \subset \mathscr{S}_{2}$, and it is locally uniformly ultimately bounded (Corless and Leitmann, 1981).

Furthermore, these regions can be characterized as follows

$$
\begin{aligned}
& \mathscr{S}_{1}=\left\{\left[\begin{array}{l}
\tilde{\xi} \\
\dot{\tilde{\xi}}
\end{array}\right]: \sum_{i=1}^{f}\left[\begin{array}{l}
\tilde{\xi}_{i} \\
\dot{\dot{\xi}} \\
{ }_{i}
\end{array}\right]^{T} \mathbf{B} \mathrm{P}_{o} \mathbf{B}\left[\begin{array}{l}
\tilde{\xi}_{i} \\
\dot{\tilde{\xi}}_{i}
\end{array}\right]<R_{1}^{2}\right\} \\
& \mathscr{S}_{2}=\left\{\left[\begin{array}{l}
\tilde{\xi} \\
\dot{\tilde{\xi}}
\end{array}\right]: \sum_{i=1}^{f}\left[\begin{array}{l}
\tilde{\xi}_{i} \\
\dot{\dot{\xi}} \\
\tilde{\xi}_{i}
\end{array}\right]^{T} \mathbf{B} \mathrm{P}_{o} \mathbf{B}\left[\begin{array}{l}
\tilde{\xi}_{i} \\
\dot{\tilde{\xi}}_{i}
\end{array}\right]<R_{2}^{2}\right\}
\end{aligned}
$$

where $\mathbf{B}=\operatorname{diag}(\beta, 1)$ and

$$
\begin{aligned}
& R_{1}= \frac{\sigma_{m}^{2} \tilde{\beta}-\sigma_{m} \sqrt{\left(\sigma_{m} \tilde{\beta}\right)^{2}-4 v \sigma_{T_{M}} \chi_{M}}}{2 v} \\
& R_{2}=\frac{\sigma_{m}^{2} \tilde{\beta}+\sigma_{m} \sqrt{\left(\sigma_{m} \tilde{\beta}\right)^{2}-4 v \sigma_{T_{M}} \chi_{M}}}{2 v}
\end{aligned}
$$

See Appendix for the proof of both theorems.

\section{NUMERICAL SIMULATIONS}

A two link planar manipulator with a camera attached to the arm tip is here considered in order to test the visual servo controller (in the following this manipulator will be called SLAVE). The manipulator is composed of two rigid arms of $0.4 \mathrm{~m}$ length each. The camera is characterized by a focal length $\lambda$ of $8 \mathrm{~mm}$ and an averaged scale factor of $100 \mathrm{pixel} / \mathrm{mm}$. The target is made up of two points, lying at the extremes of a $12 \mathrm{~cm}$ length segment, and is fixed at the arm tip of a two link planar manipulator that has the same mechanical specifications of the SLAVE (in the following this manipulator will be called MASTER). The MASTER and the SLAVE manipulators move on two parallel planes at a distance of $80 \mathrm{~cm}$.

The performance of the visual servo control law (2) and of the simplified control law (5) are here compared. Both the MASTER and the SLAVE manipulators start from an outstretched position and the MASTER 
describes a trajectory in joint space, characterized by the following fifth order polynomial relation

$$
q_{M}(t)=\left(q_{f}-q_{o}\right) \frac{t^{3}}{T^{3}}\left[10-15 \frac{t}{T}+6 \frac{t^{2}}{T^{2}}\right]
$$

where $q_{o}=\left(-60^{\circ}-30^{\circ}\right)^{T}$ and $q_{f}=\left(60^{\circ} 30^{\circ}\right)^{T}$ are the initial and final angle, respectively. The duration $T$ of the maneuver varies from $40 s$ to $5 s$, giving rise to an increase of the target acceleration and, consequently, of the joint velocities.

Although in the simplified control law (5) the target acceleration has been neglected, Fig. 2 shows that the ratio between the tip position error obtained using the visual control law (2) (dash-dot line) and the simplified control law (5) (dashed line) does not significantly increase.
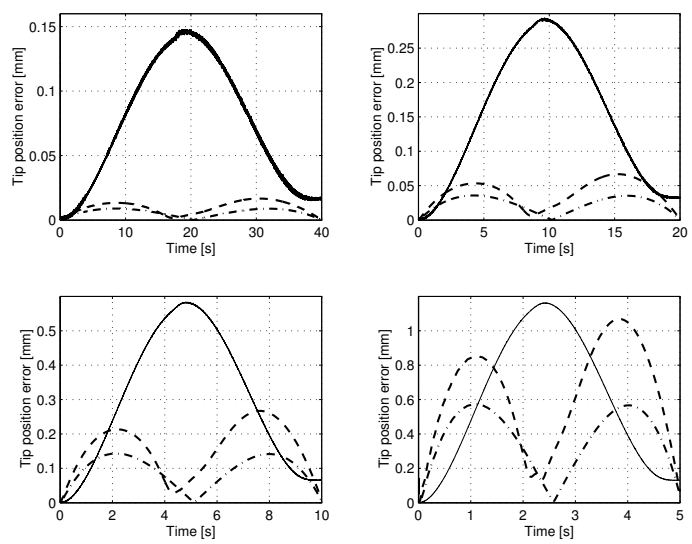

Figure 2. Tip position errors for increasing target acceleration (the solid line is the tip position error resulting from a standard task space computed torque control law, when the direct kinematic is affected by a $10 \%$ error on the link length).

\section{CONCLUSIONS}

In this paper a visual servo controller inspired by the task space computed torque control method has been proposed. In order to reduce the computational burden of this control law, two simplified controllers have been presented, supported by a stability analysis based on the Lyapunov theory. Simulation results, obtained on the model of a two link planar manipulator and a target made of two object feature points, showed the effectiveness of the proposed simplified visual servo controllers.

\section{APPENDIX}

The following preliminary results are first given, omitting the proofs.

Lemma 1. Matrix $\mathscr{A}+\beta I_{2 f}$ is Hurwitz $\forall \beta$ provided that parameters $\kappa_{01}$ and $\kappa_{02}$ satisfy conditions (8).
Lemma 2. Consider the transfer function

$$
T_{\beta}(s)=-B^{T} A\left[s I-\left(A+\beta I_{2 f}\right)\right]^{-1} B
$$

Then

$$
\left\|T_{\beta}(s)\right\|_{\infty}=\|t(s)\|_{\infty}=\left\|\mathrm{B}^{T} \mathrm{~A}_{\circ}\left[s I-\left(\mathrm{A}_{o}+I_{2}\right)\right]^{-1} \mathrm{~B}\right\|_{\infty}
$$

Lemma 3. The pair $\left(\mathscr{A}+\beta I_{2 f}, \mathscr{C}\right)$, with $\mathscr{C}=\mathscr{B}^{T} \mathscr{A}$, is observable if and only if $\kappa_{01} \neq 0$.

Lemma 4. The solution of the algebraic Riccati equation

$$
\begin{aligned}
\left(\mathscr{A}+\beta I_{2 f}\right)^{T} P+P\left(\mathscr{A}+\beta I_{2 f}\right) & +\gamma^{2} P \mathscr{B} \mathscr{B}^{T} P \\
& +\mathscr{C}^{T} \mathscr{C}=0
\end{aligned}
$$

with $C=\mathscr{B}^{T} \mathscr{A}$, may be written in the following form

$$
P=b \operatorname{diag}(\mathscr{P}, \mathscr{P}, \ldots, \mathscr{P})
$$

with $\mathscr{P}=\beta \mathbf{B P}_{o} \mathbf{B}$, where $\mathbf{B}=\operatorname{diag}(\beta, 1)$ and $\mathrm{P}_{o}$ is a solution of the algebraic Riccati equation (9).

Lemma 5. If $\|t(s)\|_{\infty}<\gamma^{-1}$, there exists the positive semidefinite stabilizing solution $\mathrm{P}_{o}$ of the Riccati equation (9) and its eigenvalues are larger than 1, i.e. $\lambda_{i}\left(\mathrm{P}_{o}\right)>1 \forall i$. Moreover if $\left(\mathscr{A}+\beta I_{2 f}, \mathscr{C}\right)$ is observable the solution is positive definite.

Proof of the Theorems: As previously mentioned, the feature error system (6) can be rearranged, through a suitable change of coordinates, into $f$ independent subsystems, whose state matrices are given by (7). Thus in the following, for the sake of simplicity, the diagonal system of state vector $\mathscr{E}=\left(\mathscr{E}_{1} \ldots \mathscr{E}_{f}^{T}\right)^{T}$, with $\mathscr{E}_{i}^{T}=\left(\tilde{\xi}_{i} \dot{\tilde{\xi}}_{i}\right)$, and state matrices $(\mathscr{A}, \mathscr{B})$ will be considered.

With the hypotheses of the theorem, from Lemma 1 and 3 it follows that matrix $\mathscr{A}+\beta I_{2 f}$ is Hurwitz and the pair $\left(\mathscr{A}+\beta I_{2 f}, \mathscr{C}\right)$, with $\mathscr{C}=\mathscr{B}^{T} \mathscr{A}$, is observable $\forall \beta$. These conditions are sufficient (see Lemma 5 ), being $\left\|T_{\beta}(s)\right\|<\gamma^{-1}$, to claim the existence of a positive definite solution $P$ of the algebraic Riccati equation (14).

Consider the candidate Lyapunov function $V(\mathscr{E})=$ $\mathscr{E}^{T} P \mathscr{E}>0 \quad \forall \mathscr{E} \neq 0$. Its time derivative, along the trajectories of the system, will be

$$
\begin{aligned}
\dot{V}=\mathscr{E}^{T} & \left(P \mathscr{A}+\mathscr{A}^{T} P-\mathscr{A}^{T} \mathscr{B} \Delta^{T} \mathscr{B}^{T} P\right. \\
& \left.-P \mathscr{B} \Delta \mathscr{B}^{T} \mathscr{A}\right) \mathscr{E}+2 \mathscr{E}^{T} P \mathscr{B} \mathbf{d}
\end{aligned}
$$

where $\mathbf{d}=\mathbf{d}_{1}+\mathbf{d}_{2}$ and

$$
\mathbf{d}_{1}=-\dot{J}(\mathbf{q}, \dot{\mathbf{q}}, \xi, \dot{\xi}, Z)\left[\begin{array}{c}
\dot{\mathbf{q}} \\
\dot{\Pi}
\end{array}\right] \quad \mathbf{d}_{2}=-J_{T}(\mathbf{q}, \xi, Z) \ddot{\Pi}
$$

Taking into account that $P$ is a solution of (14) yields

$$
\begin{aligned}
\dot{V}= & -\mathscr{E}^{T}\left(2 \beta P+\gamma^{2} P \mathscr{B} \mathscr{B}^{T} P+\mathscr{A}^{T} \mathscr{B} \mathscr{B}^{T} \mathscr{A}\right. \\
& \left.\mathscr{A}^{T} \mathscr{B} \Delta^{T} \mathscr{B}^{T} P+P \mathscr{B} \Delta \mathscr{B}^{T} \mathscr{A}\right) \mathscr{E}+2 \mathscr{E}^{T} P \mathscr{B} \mathbf{d}
\end{aligned}
$$

Finally, defining

$$
\begin{aligned}
& \mathscr{L}_{1}=\mathscr{E}^{T} \Gamma \Gamma^{T} \mathscr{E} \geq 0, \forall \mathscr{E} \quad\left(\Gamma=\gamma P \mathscr{B}+\frac{\mathscr{A}^{T} \mathscr{B} \Delta^{T}}{\gamma}\right) \\
& \mathscr{L}_{2}=\left(\mathscr{B}^{T} \mathscr{A} \mathscr{E}\right)^{T}\left(I_{f}-\frac{\Delta^{T} \Delta}{\gamma^{2}}\right)\left(\mathscr{B}^{T} \mathscr{A} \mathscr{E}\right) \geq 0, \forall \mathscr{E}
\end{aligned}
$$


being $\|\Delta\| \leq \gamma$ (and therefore $\|\Delta\|^{2} / \gamma^{2}<1$ ), and

$$
\mathscr{R}=2 \beta \mathscr{E}^{T} P \mathscr{E} \quad \mathscr{D}=2 \mathscr{E}^{T} P \mathscr{B} \mathbf{d}
$$

the time derivative of the Lyapunov function can be written as

$$
\dot{V}=-\mathscr{L}_{1}-\mathscr{L}_{2}-\mathscr{R}+\mathscr{D}
$$

From Lemma 4 it follows that $P$ is a block diagonal matrix and $\mathscr{P}=\beta \mathbf{B P}_{o} \mathbf{B}$. Thus

$$
\mathscr{R}=2 \beta^{2}\left(\psi_{1}^{T} \psi_{1}+\cdots+\psi_{f}^{T} \psi_{f}\right)=2 \beta^{2}\|\psi\|^{2}
$$

where $\psi_{i}=\mathrm{P}_{o}^{1 / 2} \mathbf{B} \mathscr{E}_{i}$ and $\psi=\left(\psi_{1}^{T} \psi_{2}^{T} \ldots \psi_{f}^{T}\right)^{T}$. Moreover, for the same reasons, it follows that

$$
\begin{aligned}
\mathscr{D} & =2 \beta \psi^{T}\left[\begin{array}{cccc}
\mathrm{P}_{o}^{1 / 2} \mathrm{~B} & \mathbf{0}_{2,1} & \ldots & \mathbf{0}_{2,1} \\
\vdots & \vdots & & \vdots \\
\mathbf{0}_{2,1} & \mathbf{0}_{2,1} & \ldots & \mathrm{P}_{o}^{1 / 2} \mathrm{~B}
\end{array}\right] \mathbf{d} \\
& \leq 2 \beta\|\psi\|\left\|\mathrm{P}_{o}^{1 / 2} \mathrm{~B}\right\|\|\mathbf{d}\|
\end{aligned}
$$

Consider now the case $\mathbf{d}=\mathbf{d}_{1}$, corresponding to Theorem 1 , and $\mathbf{d}=\mathbf{d}_{1}+\mathbf{d}_{2}$, corresponding to Theorem 2 , separately.

Case 1

Taking into account Assumption 4 it follows that

$$
\mathscr{D} \leq 2 \beta \frac{v}{\sigma_{m}^{2}}\left\|\mathrm{P}_{o}^{1 / 2} \mathrm{~B}\right\|\|\psi\|^{3}
$$

where the following inequality has been used

$$
\left\|\mathscr{E}_{i}\right\| \leq\left\|\mathbf{B} \mathscr{E}_{i}\right\| \leq\left\|\mathrm{P}_{o}^{1 / 2} \mathbf{B} \mathscr{E}_{i}\right\|=\left\|\psi_{i}\right\|
$$

Applying these bounds to equation (15) yields

$$
\dot{V} \leq \frac{2 \beta}{\sigma_{m}^{2}}\left\|\mathrm{P}_{o}^{1 / 2} \mathrm{~B}\right\|\|\psi\|^{2} h(\|\psi\|)
$$

where

$$
h(\|\psi\|)=v\|\psi\|-\sigma_{m}^{2} \tilde{\beta}
$$

and $\tilde{\beta}=\beta /\left\|\mathrm{P}_{o}^{1 / 2} \mathrm{~B}\right\|$.

In order to set $\dot{V}<0$ the following condition must hold

$$
\|\psi\|<\rho=\frac{\sigma_{m}^{2}}{v} \tilde{\beta}
$$

If this holds, the origin of the state space is an asymptotically stable equilibrium point whose region of attraction includes, in view of the definition of $\psi$, the region (10) of the space $(\tilde{\xi}, \dot{\tilde{\xi}})$.

Case 2

Taking into account Assumption 4 and Assumption 5 it follows that

$$
\mathscr{D} \leq 2 \beta\left\|\mathrm{P}_{o}^{1 / 2} \mathrm{~B}\right\|\|\psi\|\left(\frac{v}{\sigma_{m}^{2}}\|\psi\|^{2}+\sigma_{T_{M}} \chi_{M}\right)
$$

where inequality (16) has been used.

Applying these bounds to equation (15) yields

$$
\dot{V} \leq \frac{2 \beta}{\sigma_{m}^{2}}\left\|\mathrm{P}_{o}^{1 / 2} \mathrm{~B}\right\|\|\psi\| h(\|\psi\|)
$$

where

$$
h(\|\psi\|)=v\|\psi\|^{2}-\sigma_{m}^{2} \tilde{\beta}\|\psi\|+\sigma_{m}^{2} \sigma_{T_{M}} \chi_{M}
$$

and $\tilde{\beta}=\beta /\left\|\mathrm{P}_{o}^{1 / 2} \mathrm{~B}\right\|$.

There exists an interval $R_{1}<\|\psi\|<R_{2}$ on the $\|\psi\|$ axis, where $R_{1}$ and $R_{2}$ are the roots of the polynomial $h$, in which the inequality $h(\|\psi\|)<0$ holds, if and only if condition (11) is satisfied. Furthermore, as $V=$ $\|\psi\|^{2}$, the level surfaces of the candidate Lyapunov function are balls around the origin of the $z$-space. As a consequence $\exists T>t_{0}:\left\|\psi\left(t_{0}\right)\right\|<R_{2} \Rightarrow\|\psi(t)\|<$ $R_{1}, \forall t>T$. In fact, as the Lyapunov function decreases for $R_{1}<\|\psi\|<R_{2}$, being its time derivative bounded by a negative definite function of the norm of the state, every state trajectory starting from a point inside the ball of radius $R_{2}$ will approach, in finite time, the inner ball of radius $R_{1}$ and will be trapped inside it. Thus, in view of the definition of $\psi$, it is easy to conclude that there exists $T>t_{0}$ such that

$$
\left[\begin{array}{l}
\tilde{\xi}\left(t_{0}\right) \\
\dot{\tilde{\xi}}\left(t_{0}\right)
\end{array}\right] \in \mathscr{S}_{2} \Rightarrow\left[\begin{array}{l}
\tilde{\xi}(t) \\
\dot{\tilde{\xi}}(t)
\end{array}\right] \in \mathscr{S}_{1}, \forall t>T
$$

where $\mathscr{S}_{1}$ and $\mathscr{S}_{2}$ are given by (12) and (13), respectively.

\section{REFERENCES}

Chaumette, F. (1998). Potential problems of stability and convergence in image-based and positionbased visual servoing. In: The confluence of vision and control (D. Kriegman, G. Hager and S. Morse, Eds.). Vol. 237 of Lecture Notes in Control and Information Sciences. pp. 66-78. Springer-Verlag.

Corless, M. J. and G. Leitmann (1981). Continuous state feedback guaranteeing uniform ultimate boundedness for uncertain dynamic systems. IEEE Transaction on Automatic Control AC-26, 1139-1144.

Horn, B. K. P. (1986). Robot vision. MIT Press. Cambridge, Massachusetts.

Hutchinson, S. A., G. D. Hager and P. I. Corke (1996). A tutorial on visual servo control. IEEE Transactions on Robotics and Automation 12(5), 651670.

Kelly, R. (1996). Robust asymptotically stable visual servoing of planar robots. IEEE Transactions on Robotics and Automation 12(5), 759-766.

Kelly, R., R. Carelli, O. Nasisi, B. Kuchen and F. Reyes (2000). Stable visual servoing of camera-in-hand robotic systems. IEEE/ASME Transactions on Mechatronics 5(1), 39-48.

Nakamura, Y. and H. Hanafusa (1986). Inverse kinematic solution with singularity robustness for robot manipulator control. ASME Journal on Dynamic Systems, Measurements and Control 108, 163-171.

Sciavicco, L. and B. Siciliano (2000). Modeling and control of robot manipulators. 2nd ed.. SpringerVerlag. London.

Wampler, C. W. (1986). Manipulator inverse kinematic solutions based on vector formulations and damped least-squares methods. IEEE Transactions on System, Man, Cybernetics 16, 93-101. 